\title{
Application of Postprocessing Techniques Methods for Noise Reduction in Cylindrical Near Field Antenna Measurements
}

\author{
Francisco Cano Fácila, Manuel Sierra-Castañer \\ ETSI Telecomunicación, Dpto. SSR \\ Universidad Politécnica de Madrid \\ 28040 Madrid, Spain \\ francisco@gr.ssr.upm.es, mscastaner@gr.ssr.upm.es
}

\begin{abstract}
Three different methods to reduce the noise power in the far-field pattern of an antenna when it is measured in a cylindrical near field system are presented and compared. The first one is based on a modal filtering while the other two are based on spatial filtering, either on an antenna plane or either on a cylinder of smaller radius. Simulated and measured results will be presented.
\end{abstract}

Keywords-component; AWGN, antenna measurements, filtering, modal analysis, noise cancellation, reconstruction algorithms, signal-to-noise ratio.

\section{INTRODUCTION}

The objective of this paper is to describe different methods to improve the signal-to-noise ratio in the far-field pattern by reducing the noise power, focusing on random near-field errors. These errors are always present and are commonly introduced by the receiver additive noise. In many cases, these errors are negligible in the overall measurement uncertainty thanks to the use of modern receivers and sufficient amplification in the system. However, when measuring ultralow sidelobe or high performance antennas, noise may significantly alter the radiation pattern and it has to be taken into account in the near-field error budget. Some comprehensive studies for random noise in near-field measurements have already been presented. For the planar system, two independent analyses with similar results have been described in [1], [2]. Both of them start with random errors in planar near-field (PNF) and obtain expressions that represent the signal-to-noise ratio in far-field as a function of the noise power in near-field. A similar study for cylindrical near-field (CNF) measurements was carried out in [3], [4].

The reduction of noise achieved with these methods is obtained by representing the acquired near-field data in a convenient domain where it is possible to filter out a portion of noise. This idea of reducing the noise by means of a filtering in an auxiliary domain was proposed in [5], [6] for the spherical near-field (SNF) case. The same idea was used in [7] to improve the signal-to-noise ratio in the far-field pattern of an antenna as measured in PNF. In this last case, however, a spatial filtering on the antenna under test (AUT) plane is employed instead of a modal filtering. In the present work,

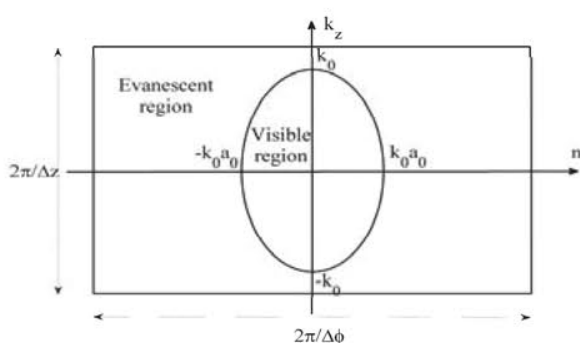

Figure 1. Evanescent region and visible region in the $\mathrm{CMC}$ domain

three different methods to reduce the noise power when measuring an antenna in CNF are proposed. All these methods are based on the a priori knowledge of the information in a different domain respect the acquisition data: cylindrical modes, antenna under test area or a smaller cylinder. In these new domains some filters can be applied reducing the noise without losing information from the AUT. Moreover, the results of a statistical analysis are presented for each case in order to determine the signal-to-noise ratio improvement.

\section{MODAL FILTERING METHOD}

As it is well-known, the initial step to determine the far-field pattern from CNF measurements is to calculate two sets of cylindrical modal coefficients (CMC) [8]. These coefficients are related to the measured field and are band-limited in the $n-k_{z}$ domain, being negligible outside of the visible region defined by

$$
n^{2}+\left(k_{z} a_{0}\right)^{2}<\left(k_{0} a_{0}\right)^{2}
$$

where $a_{0}$ is the radius of the smallest sphere enclosing the AUT (Figure 1). Therefore a filtering of the modes out of these region implies a reduction of the noise without losing information of the AUT. If the separation between samples is smaller than the values indicated in (2), more CMC are obtained in the evanescent region, where theoretically have to be negligible. However, due to the presence of undesired contributions, like reflections, diffractions, leakage signals, 
etc., the calculated CMC may be non-zero outside of the visible region. Therefore, one alternative to cancel the unwanted effects of these contributions is to apply a modal filtering, setting to zero all CMC in the evanescent region [9]. The first noise reduction method proposed in this paper is based on the same principle. An oversampling is applied in order to obtain more $\mathrm{CMC}$ than required. After that, the evanescent modes are filtered out, removing the noise contribution in this region. Finally, the filtered CMC are used to calculate a far-field pattern with less noise power.

$2 k_{0} \leq\left.\frac{2 \pi}{\Delta z} \Rightarrow \Delta z\right|_{\max }=\frac{\lambda}{2} ; 2 k_{0} a_{0} \leq\left.\frac{2 \pi}{\Delta \phi} \Rightarrow \Delta \phi\right|_{\max }=\frac{\lambda}{2 a_{0}}$

Next, simpler approximate expressions for the improvement achieved with this first filtering method are derived. Assuming that the argument values of the Hankel function and the derivate of the Hankel function are very large, the asymptotic expansion of these functions can be used instead. This condition is better fulfilled around $\theta=90^{\circ}$ where $\kappa \cong k_{0}$. Moreover, the validity region of a CNF measurement is also around the horizontal direction. Therefore, the better approximation is given for the region of interest. Thus, the improvement of the signal to noise ratio can be depicted as:

$$
\begin{gathered}
\Delta S N R_{E_{\phi}}(\theta)=\frac{\sum_{n=-N_{\phi} / 2}^{N_{\phi} / 2} \frac{n^{2} k_{z}^{2}+a^{2} \kappa^{4}}{\frac{2 \kappa^{4} a}{\pi \kappa}}}{\sum_{n=-N(\theta)}^{N(\theta)} \frac{n^{2} k_{z}^{2}+a^{2} \kappa^{4}}{\frac{2 \kappa^{4} a}{\pi \kappa}} \cong \frac{N_{\phi}}{2 N(\theta)}} \\
\Delta S N R_{E_{\theta}}(\theta)=\frac{\sum_{n=-N_{\phi} / 2}^{N} \frac{\pi \kappa a}{2}}{\sum_{n=-N(\theta)}^{N(\theta)} \frac{\pi \kappa a}{2}}=\frac{N_{\phi}}{2 N(\theta)}
\end{gathered}
$$

where $\kappa=\sqrt{k_{0}-k_{z}}, N_{\phi}$ is the number of samples in $\phi$ direction and $N(\theta)$ can be deduced from (1):

$$
N(\theta)=\sqrt{\left(k_{0} a_{0}\right)^{2}-\left(k_{z} a_{0}\right)^{2}}=k_{0} a_{0} \sqrt{1-\cos ^{2}(\theta)}=k_{0} a_{0} \sin (\theta) .
$$

Therefore, the improvement in both components is the same and is related to the sampling spacing in $\phi$ as follows

$$
\Delta S N R(\theta)=\frac{N_{\phi}}{2 N(\theta)}=\frac{\lambda}{2 \Delta \phi a_{0} \sin (\theta)}=\frac{\left.\Delta \phi\right|_{\max }}{\Delta \phi \sin (\theta)}=\frac{f_{o v, \phi}}{\sin (\theta)}
$$

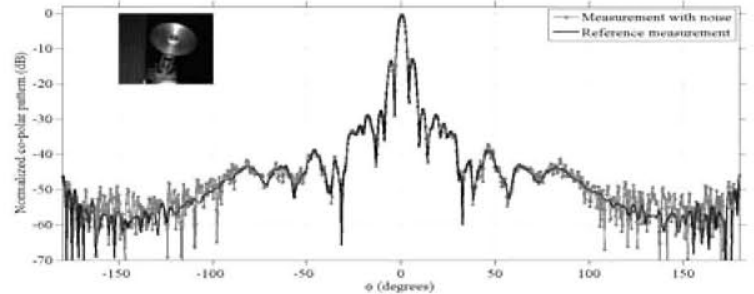

(a)

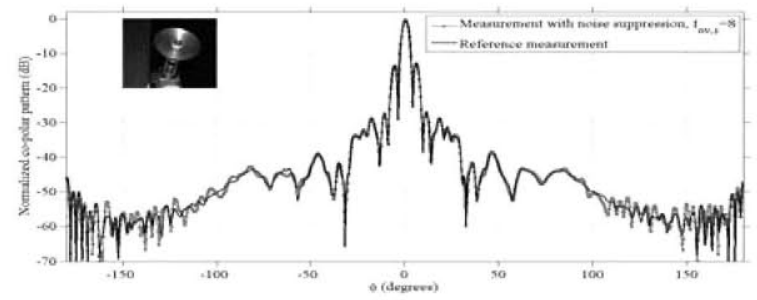

(b)

Figure 2. Far-field comparison between (a) the reference pattern and the pattern with noise and (b) the reference pattern and the pattern after modal filtering with an oversampling factor equal to 8 in the horizontal plane.

where it is deduced that the improvement is directly proportional to $f_{o v, \phi}$, which is the oversampling factor in azimuth, and increases when we move away from the horizontal direction.

This method is validated using information from a measurement in the cylindrical near-field range of the Technical University of Madrid (UPM). For the experiment, the probe and the AUT consisted of a corrugated conical-horn antenna and a $\mathrm{Ku}$-band reflector $(14 \mathrm{GHz})$, with a $40 \mathrm{~cm}$ diameter, respectively. The data were acquired over a cylinder with a height of $1.8 \mathrm{~m}$ and a radius of $0.63 \mathrm{~m}$ and with a spatial sampling equal to $0.5 \lambda$ in the vertical direction and $0.375^{\circ}$ in azimuth. Because the maximum sampling spacing in azimuth is $3.06^{\circ}$, the oversampling factor was approximately 8. After measuring the AUT, Gaussian noise with $42 \mathrm{~dB}$ less power than the maximum of the acquired data was computationally added. The noise power was chosen to be large, so as to ensure a negligible measurement noise. The farfield obtained from the measured data without additive noise can thus be used as a reference to compare results before and after noise filtering. The far-field results obtained before and after applying the filtering are compared with the reference pattern in Fig. 2 and the average improvement is $9.61 \mathrm{~dB}$.

\section{Spatial Filtering Method on The AUT Plane}

A second method to reduce the noise power in the farfield pattern obtained from a CNF measurement is presented in this section. As in the previous method, a field transformation that allows filtering out a portion of noise without modifying the desired signal is required. The transformation used here was already proposed in [7] for noise reduction in far-field results obtained from PNF measurements and consists of back-propagating the measured field from the scan surface to the plane of the AUT (Figure 3). In this new 


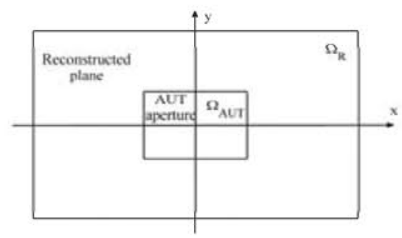

Figure 3. AUT area and reconstructed plane for second method.

domain, the desired contribution is theoretically concentrated in the antenna aperture, whereas the noise is spread over the whole reconstructed surface. Thanks to this fact, a spatial filtering may be applied, canceling the field that is located out of the AUT dimensions and that is only composed of noise.

It can be deduced from a statistical analysis that the noise in the extreme near-field is correlated both in $x$ and $y$. However, all the samples have the same noise power (autocorrelation for $\chi=\xi=0$ ), i.e., the noise is stationary and is identically distributed over the whole reconstructed surface, whereas the desired field is theoretically concentrated in the aperture antenna. Therefore, a spatial filtering may be applied, canceling the field that is located out of the AUT dimensions and that is only composed by noise. This spatial filtering is defined in (7).

$$
G(x, y)= \begin{cases}1 & \Omega_{A U T} \\ 0 & \Omega_{R}-\Omega_{\mathrm{AUT}}\end{cases}
$$

Because the spatial filtering does not modify the desired information (located within the AUT aperture), the last result can be employed to determine the average signal-to-noise ratio improvement achieved with this second method.

$$
\Delta S N R_{A V, \text { spatial }}=\frac{S_{\Omega_{R}}}{S_{\Omega_{A U T}}}
$$

where $S_{\Omega_{R}}$ and $S_{\Omega_{A U T}}$ symbolize the area of the reconstructed plane and AUT aperture, respectively. Although it seems we can obtain an infinite improvement by increasing the size of the reconstructed surface, there is a limitation that is related to the interpolation required in the process. A larger reconstructed surface implies a larger interpolation. Therefore, the PWS samples are more correlated and the previous analysis is no longer valid.

The spatial filtering method was validated by employing the data from the actual measurement described before. The only changes were, as in the previous example, the noise power, that in this case was $35 \mathrm{~dB}$ less than the maximum of the measured data, and the sampling spacing in phi, that was equal to $1.5^{\circ}$. The reconstructed surface employed in this case was a $1.8 \mathrm{~m} \times 1.8 \mathrm{~m}$ plane. Because the AUT was a reflector of 0.4 m diameter, the theoretical improvement was $14.11 \mathrm{~dB}$, but

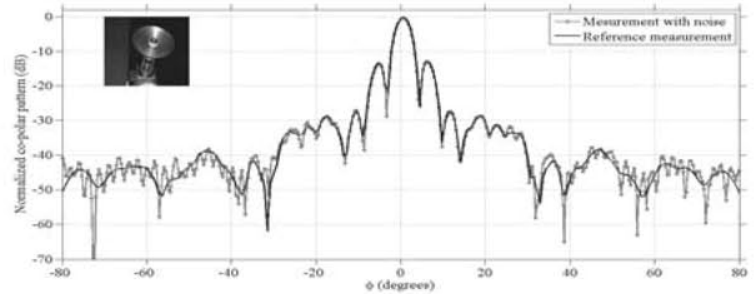

(a)

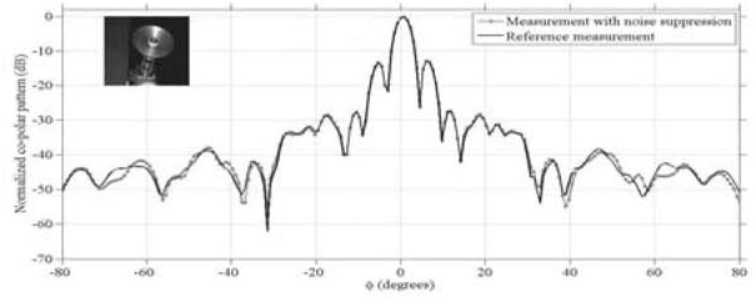

(b)

Figure 4. Far-field comparison between (a) the reference pattern and the pattern with noise, (b) the reference pattern and the pattern after spatial filtering in the horizontal plane.

due to the interpolation process, the observed improvement was $13.29 \mathrm{~dB}$. Figure 4 depicts the results, showing that it was possible to retrieve the reference pattern with good accuracy. Although this second method achieves a larger improvement using less measured data, the noise is only reduce in the forward hemisphere.

\section{SPATIAL FILTERING IN A SMALLER RADIUS CYLINDER}

In order to obtain the values of the CMC in the whole visible region, it is necessary to measure on an infinite cylinder. However, this is never possible, and therefore, the $\mathrm{CMC}$ obtained from the measured data are also negligible outside of the so-called validity region. Due to this limitation, the far-field pattern cannot be determined in those directions where out of that region. This is the well-known truncation error of the CNF measurement systems. Moreover, when back-propagating the measured field from the measurement cylinder to a cylinder of smaller radius, the AUT contribution is not spread over the whole cylinder, but over a smaller surface because, if the field that we can determine from the $\mathrm{CMC}$ is limited in elevation. This effect of concentration of the field can be observed in Figure 5, where the field measured over a cylindrical surface has been back-propagated to a smaller radius cylinder.

When back-propagating the field from the measurement cylinder to a smaller radius cylinder, the noise is correlated both in $\phi$ and $z$ because the autocorrelation is nonzero for $\chi \neq 0$ and $\gamma \neq 0$, respectively. Moreover, the noise variance (autocorrelation for $\chi=\gamma=0$ ) in the new surface is directly proportional to the noise variance in the measurement surface and it does not depend on the coordinates $\phi$ and $z$, i.e., the noise is stationary, and therefore, the noise power is uniformly spread in the new surface. Due to this fact, a spatial filtering may be applied to suppress the noise located out of the region where the desired 


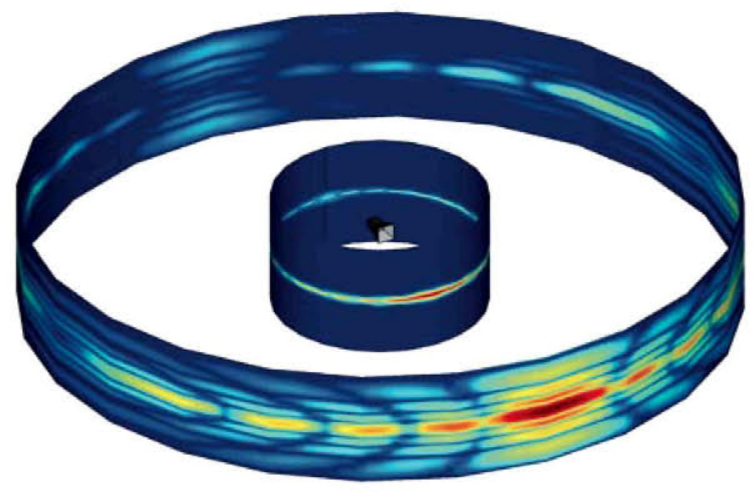

Figure 5. Example of cylindrical acquisition in two cylinders.

field is concentrated. The extension of this region was studied, reaching the conclusion that it is a horizontal strip within the cylinder where the field has been back-propagated to and with a height of $L_{2}$ defined in Figure 6. Therefore, the filtering that can be applied is as indicated in (9):

$$
W(z)= \begin{cases}1 & |z|<\frac{L_{2}}{2} \\ 0 & |z| \geq \frac{L_{2}}{2}\end{cases}
$$

In conclusion, if the field is back-propagated from the measurement cylinder to a smaller radius cylinder, a spatial filtering can be applied to suppress a portion of noise. This filtering is able to reduce the noise power both in the modal domain and in the far-field pattern by a factor $L_{2} / L_{1}$. Moreover, the filtering does not modify the desired information coming from the AUT. Therefore, the signal to noise ratio improvement achieved with this method can be calculated as the ratio between the noise power before and after applying the filtering, which is always larger than 1.

$$
\begin{gathered}
\Delta S N R_{E_{\phi}}=\frac{\sigma_{E_{\phi}^{A W G N}(\theta, \phi)}^{2}}{\sigma_{E_{\phi, f l t}^{A W G N}(\theta, \phi)}^{2}}=\frac{L_{1}}{L_{2}} \\
\Delta S N R_{E_{\theta}}=\frac{\sigma_{E_{\theta}^{A I W G N}(\theta, \phi)}^{2}}{\sigma_{E_{\theta, f i t}^{A W G N}(\theta, \phi)}^{2}}=\frac{L_{1}}{L_{2}}
\end{gathered}
$$

The spatial filtering method was validated by employing the data from the actual measurement described before (Figure 7). In this case, the noise power was $39 \mathrm{~dB}$ lower than the maximum of the measured data, and the sampling spacing in phi was equal to $2.5^{\circ}$ and the sampling space in $\mathrm{z}$ was equal to $1 \mathrm{~cm}$. The height of the measurement cylinder was $180 \mathrm{~cm}$, the radius of the measurement cylinder was $63 \mathrm{~cm}$ and the radius of the smaller cylinder was $20 \mathrm{~cm}$. In this case, the improvement in signal to noise ratio is $3.29 \mathrm{~dB}$, because of

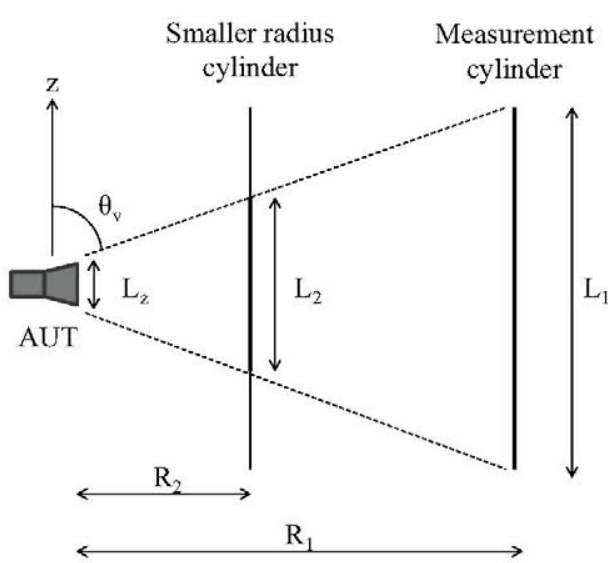

Figure 6. Back propagation of the field to a smaller radius cylinder.

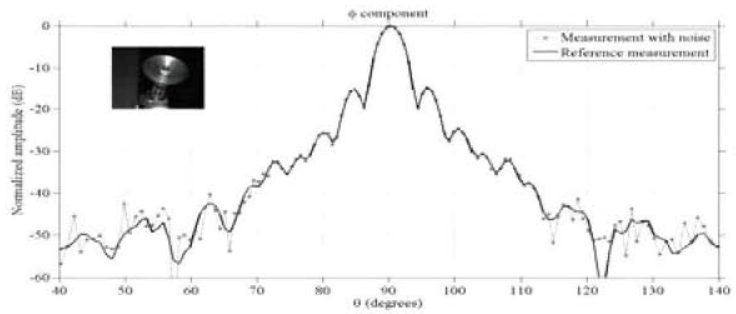

(a)

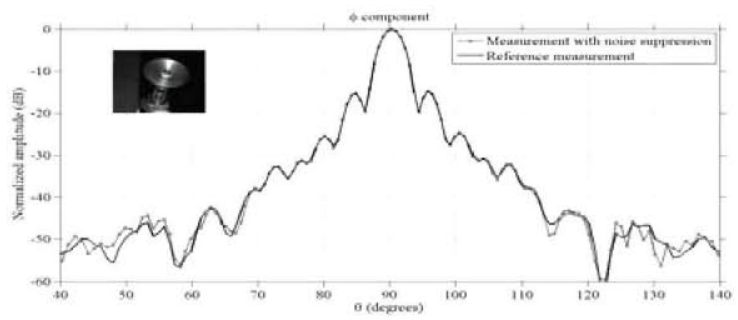

(b)

Figure 7. Far-field comparison between (a) the reference pattern and the pattern with noise, (b) the reference pattern and the pattern after spatial filtering in a smaller radius cylinder.

that the antenna was measured at a small distance.

\section{CONCLUSIONS}

Three simple and efficient methods to improve the signalto-noise ratio in the far-field results obtained from cylindrical near-field measurements were presented. The first method applies a filtering in the modal domain and its main drawback is the necessity of oversampling, increasing the measurement time. However, this is not a critical aspect when measuring in a multi-probe system. Moreover, it is possible to improve the signal-to-noise ratio in all directions. The second method employs a source reconstruction technique to filter out the noise in the extreme near-field. Unlike the modal filtering method, this second approach does not require an oversampling. In addition, a better improvement is obtained using less measured data. However, this method only provides a noise reduction in the forward hemisphere. Approximate theoretical expressions were derived for the improvement achieved with both methods. The third method uses a 
reconstruction in a cylinder of smaller radius than the measurement one. Then, a spatial filtering is realized. In this case, the gain depends on the geometry of the measurement configuration and the AUT.

Simplified expressions for the gain in SNR for the three methods were depicted in the paper. Finally, these methods were verified through application to measured near-field data of a reflector antenna in the Cylindrical Near field system of the Technical University of Madrid.

\section{ACKNOWLEDGEMENTS}

This work developed in this chapter has been realized under the project CCG10-UPM_TIC-5805, supported by Comunidad de Madrid and Universidad Politécnica de Madrid.

\section{REFERENCES}

[1] A. C. Newell and C. F. Stubenrauch, "Effect of random errors in planar near-field measurement," IEEE Trans. Antennas Propagat., vol. 36, No. 6, pp. 769-773, Jun., 1988.

[2] J. B. Hoffman and K. R. Grimm, "Far-field uncertainty due to random near-field measurement error," IEEE Trans. Antennas Propagat., vol. 36, No. 6, pp. 774-780, Jun., 1988.
[3] J. Romeu, L. Jofre, and A. Cardama, "Far-field errors due to random noise in cylindrical near-field measurements," IEEE Trans. Antennas Propagat., vol. 40, No. 1, pp. 79-84, Jan., 1992.

[4] J. Romeu and L. Jofre, "Effect of random errors in cylindrical near-field measurements," in Proc. 1991 IEEE/AP-S Int. Symp., London, ON, Jun. 24-28, 1991, pp. 1450-1453.

[5] P. Koivisto, "Reduction of errors in antenna radiation patterns using optimally truncated spherical wave expansion," Progress In Electromagnetic Research, vol. pier-47, pp. 313-333, 2004.

[6] L. J. Foged and M. Faliero, "Random noise in spherical near-field systems," in Proc. 2009 Antenna Meaurement. Techniques Assoc., AMTA, Salt Lake City, UT, Nov. 1-6, 2009, pp. 135-138.

[7] F. J. Cano-Fácila, S. Burgos, and M. Sierra-Castañer, "Novel method to improve the signal-to-noise ratio in the far-field results obtained from planar near-field measurements," IEEE Antennas Propagat. Mag., vol. 52, No. 2, Apr., 2011.

[8] O. M. Bucci and C. Gennarelli, "Use of sampling expansions in nearfield-far-field transformations: the cylindrical case," IEEE Trans. Antennas Propagat., vol. 36, No. 6, pp. 830-835, Jun., 1988.

[9] S. Gregson, A. Newell, and G. Hindman, "Reflection suppression in cylindrical near-field antenna measurement systems-cylindrical MARS," in Proc. 2009 Antenna Meaurement. Techniques Assoc., AMTA, Salt Lake City, UT, Nov. 2009, pp. 119-125. 